\title{
Rôle des forçages météo-marins dans l'évolution morphosédimentaire récente (1952-2005) des cordons littoraux de la rade de Brest.
}

\author{
Pierre Stéphan \\ Doctorant au laboratoire Géomer - UMR 6554 CNRS, IUEM.
}

Résumé : Dans un premier temps, l'évolution morphosédimentaire récente de dix cordons littoraux situés en rade de Brest est retracée par une méthode de traitement numérique de photographies aériennes prises par l'IGN entre 1952 et 2004 auxquelles s'ajoutent des levés DGPS (2004-2005). Puis, la chronologie précise des forçages météo-marins est reconstituée sur chacun des sites d'étude à partir des données marégraphiques et météorologiques. Enfin, les relations entre l'évolution mi-séculaire des cordons littoraux et les forçages météo-marins sont étudiées et permettent de définir trois types de réponse morphosédimentaire face aux épisodes morphogènes.

Abstract : Firstly, the morphosedimentary changes of ten gravel beaches located in the bay of Brest are analysed using aerial photographs taken between 1952 and 2004 and DGPS surveys (2004-2005). An analysis of meteorological and oceanographic conditions during this period is then carried out using tide, wind speed and direction data. Finally, the synthesis of these data shows the important role of high water levels and wind conditions in the erosion of this gravel beaches.

Mots clés : forçages météo-marins, évolution morphosédimentaire, cordons littoraux, rade de Brest.

\section{Introduction}

La rade de Brest se présente sous la forme d'un vaste plan d'eau qui communique avec la mer d'Iroise par un goulet étroit, atténuant fortement l'action des houles océaniques à l'intérieur de la rade (figure 1). Les vagues les plus morphogènes sont donc générées par le vent local et leur hauteur ne dépasse jamais 1,5 mètres en raison des faibles longueurs de fetch. L'hydrodynamisme réduit qui caractérise ce secteur a néanmoins permis l'édification d'une trentaine de cordons littoraux d'une diversité morphologique exceptionnelle à l'échelle du littoral atlantique français ${ }^{[2]}$ (figure 2). Ces derniers sont constitués de sédiments grossiers issus de l'érosion des falaises de schistes et du démantèlement des coulées périglaciaires. A l'exception de certains d'entre eux qui ont été largement modifiés par l'action de l'homme ${ }^{[6][7]}$, ces constructions littorales ont évolué naturellement. Elles offrent donc un terrain d'étude intéressant pour déterminer l'influence des forçages météo-marins dans les changements morphosédimentaires récents qui les caractérisent.

L'intérêt d'une telle étude est double. Il s'agit, d'une part, de réactualiser les travaux menés dans les années 1950 par A. Guilcher ${ }^{[2]}$ sur ces accumulations littorales afin de mieux comprendre leur mise en place et leur fonctionnement, et 
d'apporter d'autre part des connaissances nouvelles sur le milieu littoral de la rade de Brest, dans le cadre d'une politique de mise en valeur du patrimoine naturel menée par les collectivités territoriales et notamment par Brest Métropole Océane (BMO).

L'analyse a porté sur dix cordons littoraux situés dans la partie orientale de la rade de Brest (figure 1). Après avoir retracé leur mobilité, nous avons dressé la chronologie précise des événements morphogènes depuis 50 ans sur chacun des sites étudiés. Enfin, les relations entre l'évolution morphosédimentaire récente de ces accumulations et les épisodes morphogènes ont été étudiés.

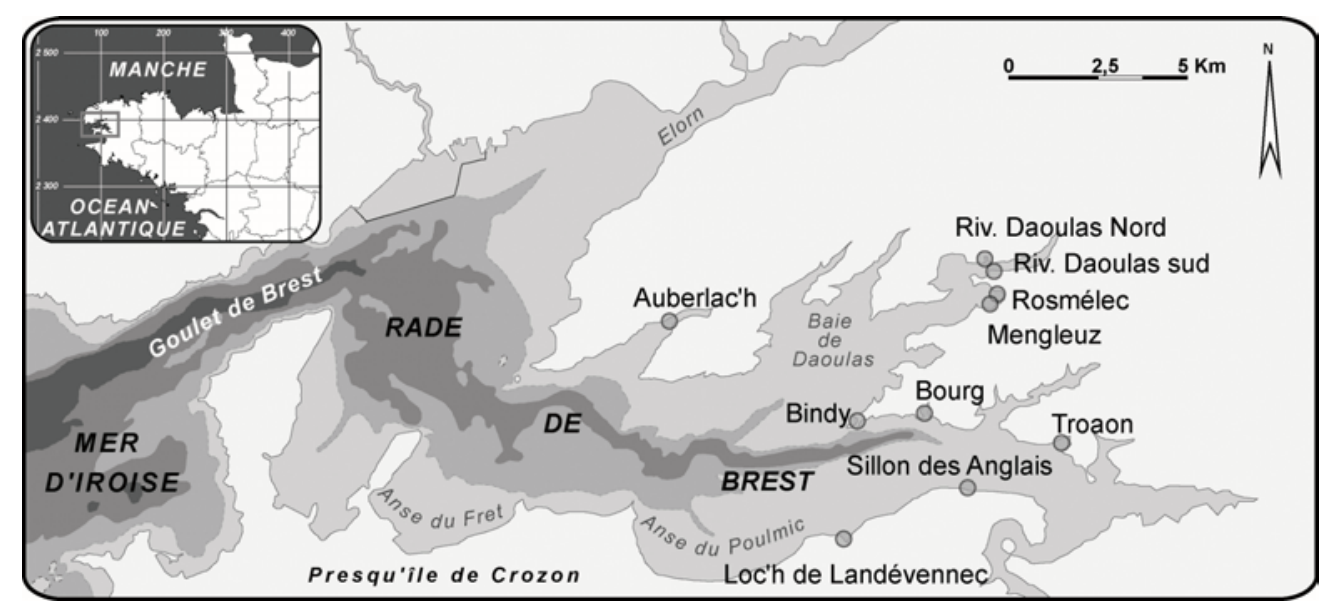

Figure 1 : Localisation des sites d'étude.
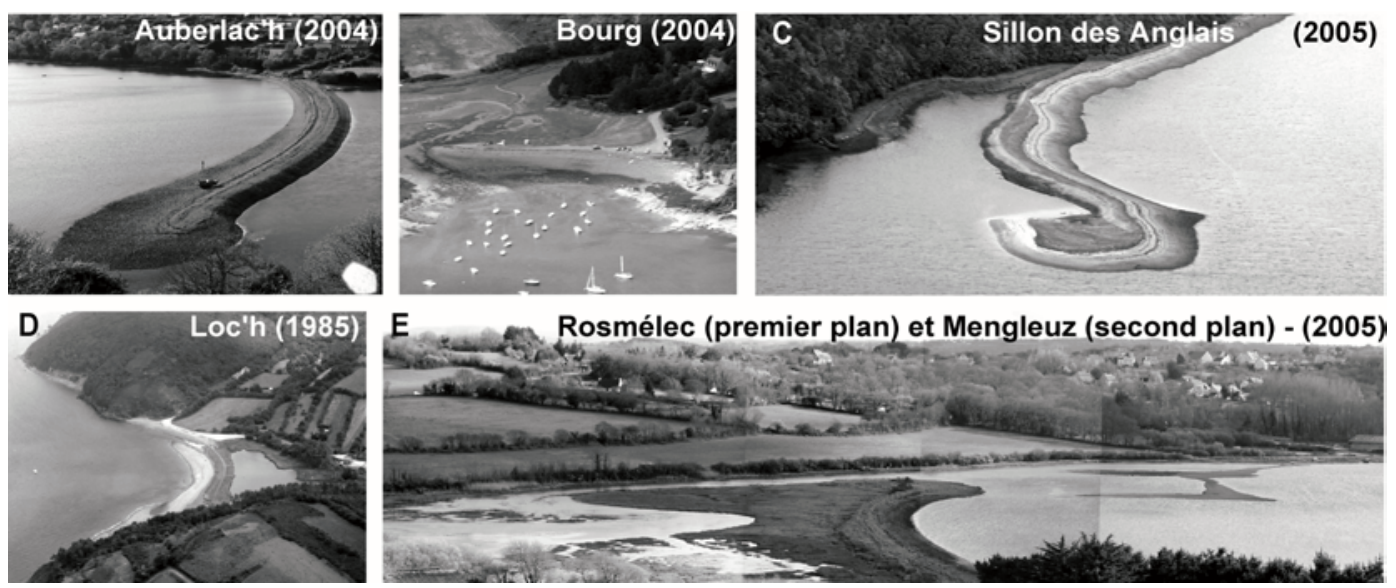

Figure 2 : Exemples de quelques cordons littoraux étudiés.

\section{La mobilité des cordons littoraux (1952-2005)}

L'évolution morphosédimentaire récente des cordons littoraux a été retracée en utilisant, d'une part des photographies aériennes prises par l'IGN entre 1952 et 2000, et d'autre part des levés effectués au DGPS en 2004 et 2005 sur quelques secteurs. Les clichés aériens ont été numérisés à très haute résolution afin d'obtenir une définition au sol de $50 \mathrm{~cm} /$ pixel. Chaque image a ensuite subi une correction géométrique avant d'être géoréférencée et intégrée dans une base SIG 
(Système d'Information Géographique) sous le logiciel Arcview 8.3. Les marges d'erreurs inhérentes à la correction géométrique et au géoréférencement des images ont été mesurées et ne dépassent pas $50 \mathrm{~cm}$ dans les zones étudiées. Enfin, la cinématique du front de végétation a été retracée en calculant les surfaces perdues ou gagnées sur chacun des cordons.

Aucune tendance générale à l'érosion ou à l'engraissement ne se dégage sur les dix cordons littoraux. Les rythmes, les vitesses et les modalités d'évolution présentent une grande variabilité spatio-temporelle interdisant toute généralisation. Il apparaît donc clairement que chaque secteur est soumis à l'influence de forçages météo-marins suivant des modalités qui lui sont propres. Cela reste essentiellement lié aux conditions d'exposition, très variables d'un site à un autre en rade de Brest.

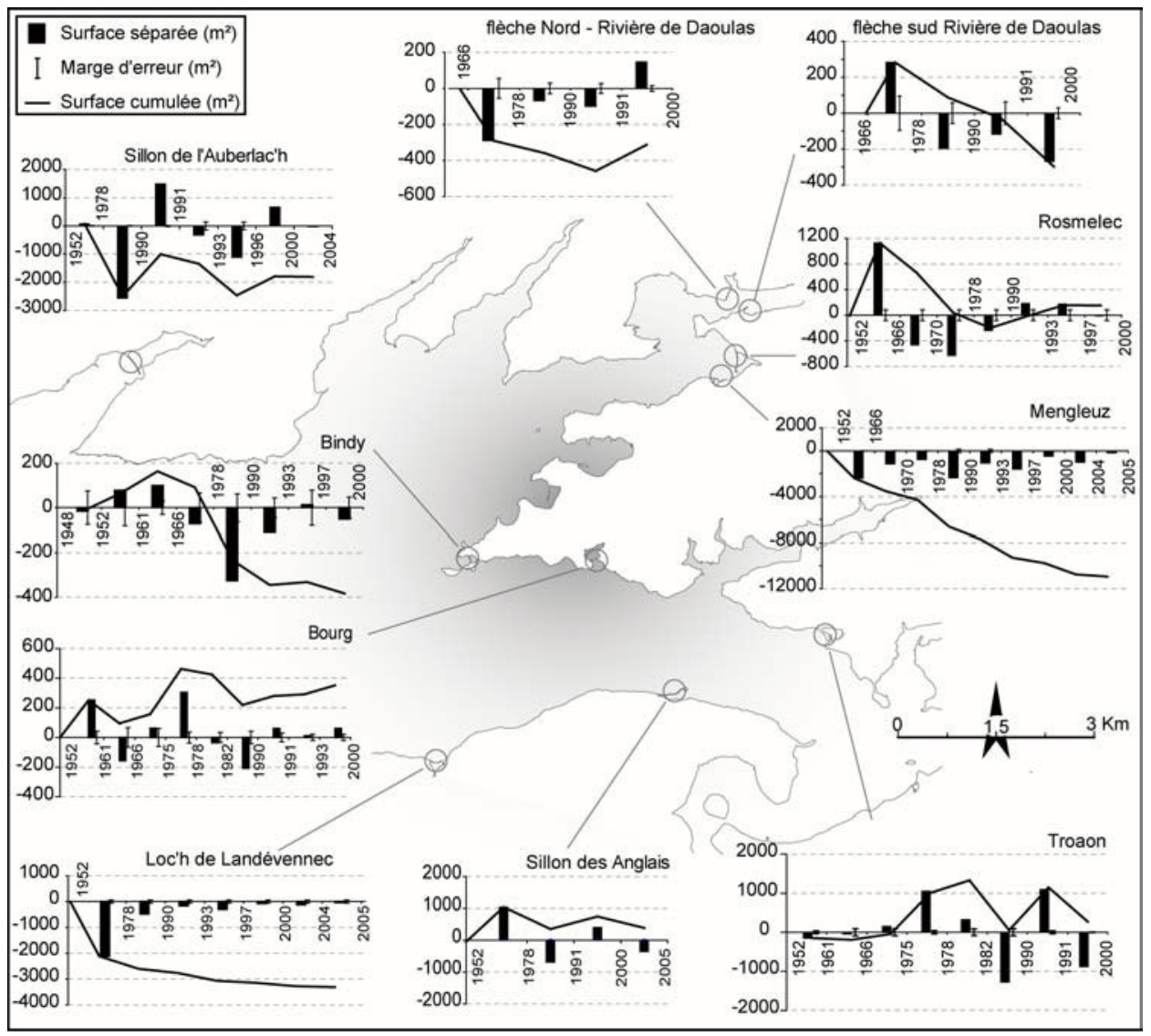

Figure 3 : Bilan surfacique (en $\mathrm{m}^{2}$ ) des cordons littoraux entre 1952 et 2004.

\section{Analyse des agents de forcages météo-marins}

En l'absence d'interventions anthropiques, seuls les plus hauts niveaux marins s'accompagnant d'une forte agitation du plan d'eau peuvent provoquer un recul des cordons littoraux. Par conséquent, pour identifier l'ensemble de ces évènements " morphogènes » sur notre période d'observation, les caractéristiques marégraphiques et hydrodynamiques ont été reconstituées entre 1952 et 2004. 
Dans un premier temps, l'altitude du front de végétation des cordons littoraux a été levée sur chacun des sites au DGPS (Figure 4).

\begin{tabular}{|l|l|l|l|}
\hline Cordons & $\begin{array}{l}\text { Altitude du front de } \\
\text { végétation (0 hydro) }\end{array}$ & Cordons & $\begin{array}{l}\text { Altitude du front de } \\
\text { végétation (0 hydro) }\end{array}$ \\
\hline Auberlac'h & $7,79 \mathrm{~m}$ & Bindy & $7,96 \mathrm{~m}$ \\
\hline Riv.Daoulas.Sud & $7,50 \mathrm{~m}$ & Bourg & $7,66 \mathrm{~m}$ \\
\hline Riv.Daoulas.Nord & $7,49 \mathrm{~m}$ & Troaon & $8,04 \mathrm{~m}$ \\
\hline Rosmélec & $7,82 \mathrm{~m}$ & Les Anglais & $7,89 \mathrm{~m}$ \\
\hline Mengleuz & $6,83 \mathrm{~m}$ & Loc'h & $7,79 \mathrm{~m}$ \\
\hline
\end{tabular}

Figure 4 : Données issues des levés DGPS.

Ensuite, la chronologie des niveaux marins extrêmes au cours desquels la mer a pu atteindre et/ou dépasser l'altitude du front de végétation a été reconstituée à partir de deux types de données :

1) les hauteurs d'eau horaires mesurées par le marégraphe de Brest (marée observée) entre 1952 et 2004 qui tiennent compte non seulement les oscillations du plan d'eau liées à la marée astronomique, mais également des phénomènes de surcote et de décote;

2) les valeurs du run-up ou $R^{T}$, c'est à dire l'élévation du niveau marin à la côte liée à l'agitation du plan d'eau, qui ont été obtenues à partir de l'équation suivante ${ }^{[5]}$ :

$R^{T}{ }_{2 \%}=0,92 H_{s} \xi_{o}$

où $R^{T}{ }_{2} \%$ correspond au run-up dépassé par $2 \%$ des plus hauts jets de rive, $H_{s}$ est la hauteur significative des vagues, $\xi_{o}$ est le nombre d'Iribarren d'équation :

$\xi_{o}=\frac{\tan \beta}{\left(H_{s} / L_{o}\right)^{1 / 2}}$

où $\tan \beta$ correspond à la pente de la plage et $\mathrm{L}_{\mathrm{o}}$ est la longueur d'onde $\left(\mathrm{gT}^{2} / 2 \pi\right)$.

Dans un second temps, l'énergie libérée par les vagues à la côte au moment de ces hauts niveaux d'eau a été calculée à partir de l'équation suivante :

$E=\frac{1}{8} \rho g H_{s}^{2}$

où E est la somme de l'énergie par unité de surface $\left(\mathrm{N} \cdot \mathrm{m}^{-2}\right), \rho$ est la densité de l'eau et $g$ est la gravité. Cette énergie est essentiellement fonction des conditions d'agitation du plan d'eau (hauteur significative $H_{s}$ et période $T_{m}$ des vagues) qui ont été reconstituées à partir des équations de Hasselmann ${ }^{[3]}$ (JONSWAP-based curves) modifiées par Vincent et Resio ${ }^{[8]}$ et qui donnent :

$$
\begin{aligned}
\frac{g H_{s}}{u_{*}^{2}} & =200 \tanh 0,003877\left(\mathrm{gd} / u_{*}^{2}\right)^{3 / 4} \times \tanh \left\{\frac{0,0002129\left(\mathrm{gF} / u_{*}^{2}\right)^{1 / 2}}{\tanh 0,003877\left(\mathrm{gd} / u_{*}^{2}\right)^{3 / 4}}\right\} \\
\frac{g T_{m}}{u_{*}} & =200 \tanh 0,07125\left(\mathrm{gd} / u_{*}^{2}\right)^{3 / 8} \times \tanh \left\{\frac{0,00426\left(\mathrm{gF} / u_{*}^{2}\right)^{1 / 3}}{\tanh 0,07125\left(\mathrm{gd} / u_{*}^{2}\right)^{3 / 8}}\right\}
\end{aligned}
$$

où $\mathrm{d}$ est la profondeur, $\mathrm{F}$ est le fetch, $\mathrm{u} *$ est la vitesse de friction calculée à partir du coefficient de résistance $c_{d}$ donné par la formule de Garratt ${ }^{[1]}$ : 
$c_{d}=10^{-3}\left(0,75+0,067 \mathrm{u}_{10}\right)$

$u_{*}=\sqrt{c_{d}} \mathrm{u}_{10}$

où $\mathrm{u}_{10}$ est la vitesse du vent mesurée à $10 \mathrm{~m}$ de la surface. Les données de force et de direction du vent utilisées dans cette étude ont été obtenues auprès de la station météorologique de Lanvéoc-Poulmic, située à $80 \mathrm{~m}$ d'altitude. Par conséquent, les vitesses de vent ont été corrigées à partir de l'équation suivante ${ }^{[8]}$ :

$\mathrm{u}_{10}=\mathrm{u}_{\mathrm{z}}\left(\frac{10}{z}\right)^{1 / 7}$

L'approche paramétrique, utilisée ici, peut être sujet à discussion car elle ne tient pas compte d'un grand nombre de facteurs complexes qui interagissent en situation réelle et peuvent modifier localement et temporairement les conditions d'agitation du plan d'eau: variations des directions de vent jouant sur la géométrie du fetch, effets de la topographie locale sur la force des vents ${ }^{[8]}$, état de développement des vagues, stratification atmosphérique ${ }^{[10]}$, phénomène de réfraction, génération d'onde interne absorbant l'énergie des vagues. Néanmoins, cette approche a été préférée à la modélisation pour des raisons d'ordre pratique : simplicité et rapidité d'utilisation.

Les résultats montrent que le cordon du Bourg et ceux situés sur les rives nord et sud de la rivière de Daoulas sont affectés par un grand nombre d'épisodes météomarins peu énergétiques (figure 5). Ces secteurs bénéficient de conditions d'abri (fetch réduit) qui empêchent le développement de vagues morphogènes $(\mathrm{E}<0,4)$. Les autres cordons étudiés présentent une situation inverse, les évènements météo-marins auxquels ils sont soumis sont moins fréquents mais beaucoup plus énergétiques (figure 6 et 7). Ces secteurs sont ouverts à de grandes longueurs de fetch, donnant naissance à des vagues particulièrement morphogènes dès lors que la force et la direction du vent le permet.

\section{Synthèse des évolutions morphosédimentaires et des conditions de forcage météo-marines}

Les figures 5, 6 et 7 mettent en relation l'évolution des cordons littoraux et les conditions de forçage météo-marines entre 1952 et 2005. Trois groupes d'individus se distinguent en fonction de leur réponse morphosédimentaire face aux évènements morphogènes.

Les cordons du Bourg et ceux de la rivière de Daoulas forment un premier groupe. Le grand nombre d'événements morphogènes peu énergétiques qui affectent ces secteurs rend difficile une quelconque interprétation des résultats. Les travaux de Jackson et al. $^{[4]}$ indiquent qu'un faible degré d'exposition des cordons littoraux induit des modalités d'évolution bien spécifiques. Se succèdent ainsi des phases d'érosion mineures et de courtes périodes de réajustement morphosédimentaire que l'on ne peut cerner à partir des photographies aériennes prises à un pas de temps pluriannuel. 


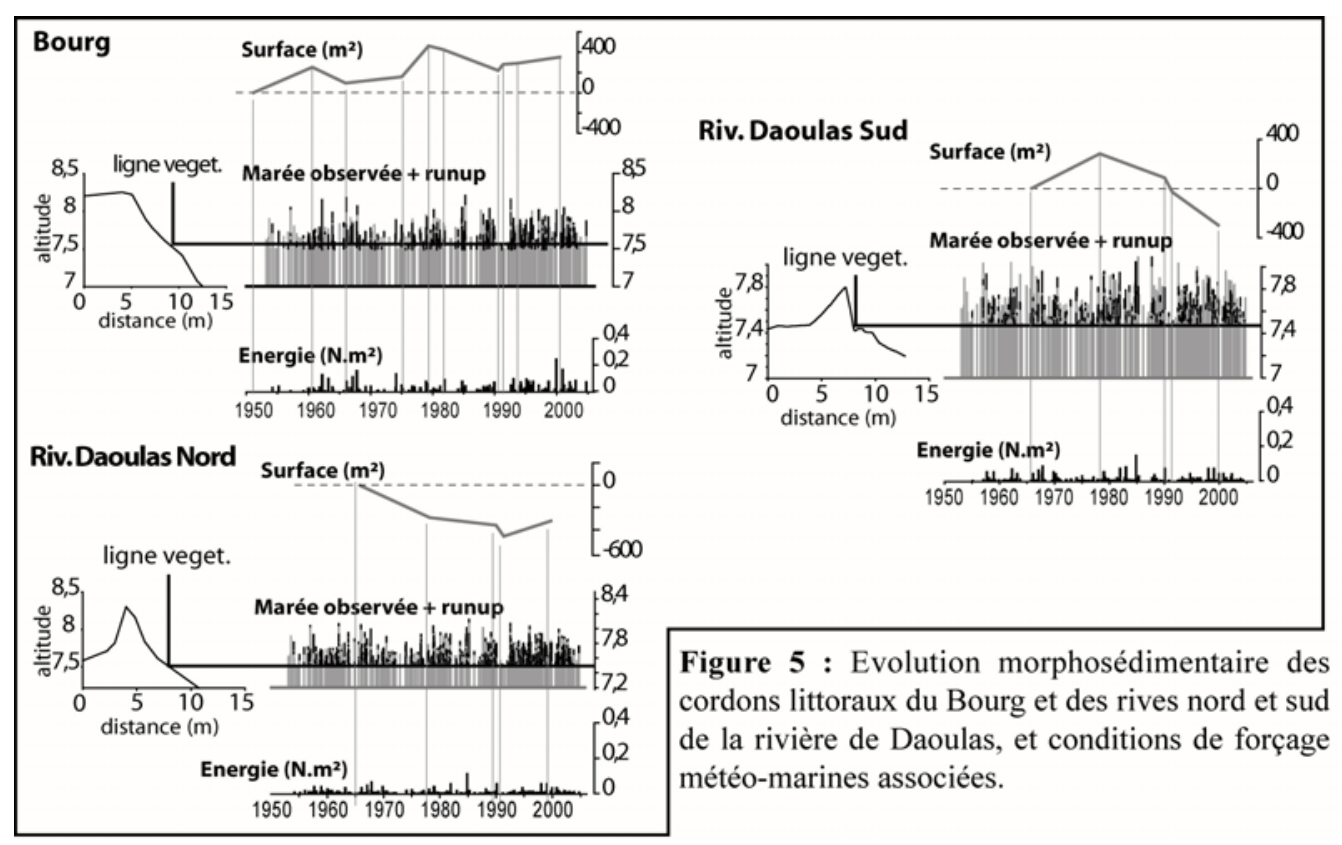

Les cordons de Mengleuz et du Loc'h composent le second groupe. Leur évolution morphosédimentaire, marquée une forte tendance au recul depuis 1952, s'effectue sans lien apparent avec les forçages météo-marins. A Mengleuz, l'érosion est liée à une situation de pénurie sédimentaire causée par les activités anthropiques ${ }^{[6]}$. Au Loc'h, cette érosion s'explique par un déséquilibre morphosédimentaire qui ne permet plus au cordon de résister aux épisodes de submersion et de forte énergie ${ }^{[7]}$.

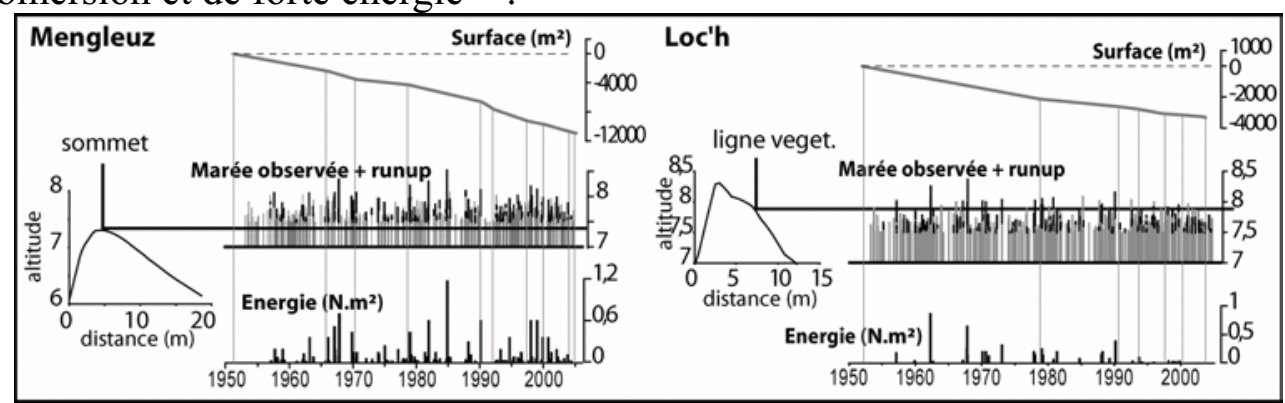

Figure 6: Evolution morphosédimentaire des cordons littoraux de Mengleuz et du Loc'h et conditions de forçage météo-marines associées.

Les cordons de l'Auberlac'h, du Bindy, de Rosmélec, de Troaon et des Anglais forme un troisième groupe. Ils sont affectés par des phases bien distinctes de recul et d'avancée, étroitement liées aux conditions de forçage météo-marines. L'érosion de ces accumulations s'effectue lors des périodes très énergétiques marquées par quelques épisodes de très hauts niveaux marins et de forte agitation du plan d'eau. Le cordon de Rosmélec illustre bien ce fonctionnement (figure 6). L'évolution de ce secteur est marquée par une phase d'érosion entre 1966 et 1990, liée à deux séries d'événements morphogènes, en novembre 1967 et en novembre 1984, au cours desquels de fortes vagues $\left(H_{s}\right.$ de $0,76 \mathrm{~m}$ et de $0,98 \mathrm{~m}$ respectivement) coïncident avec une pleine mer de vive-eau, entraînant la submersion de la ligne de végétation de $51 \mathrm{~cm}$ et de $71 \mathrm{~cm}$ respectivement, et 
taillant le cordon en une micro-falaise d'érosion marine, toujours visible dans la topographie actuelle (figure 7). Par la suite, la période 1990/1997 se marque par une avancée du cordon en l'absence d'événements morphogènes. Enfin, deux épisodes de forte énergie caractérisent la période 1997/2000 mais n'entraînent pas un recul important du cordon. Cette fois, la forte agitation du plan d'eau ne coïncide pas avec de très hauts niveaux marégraphiques et, malgré un run-up important $\left(R_{2 \%}^{T}=41 \mathrm{~cm}\right)$, la submersion est réduite $(16 \mathrm{~cm})$.

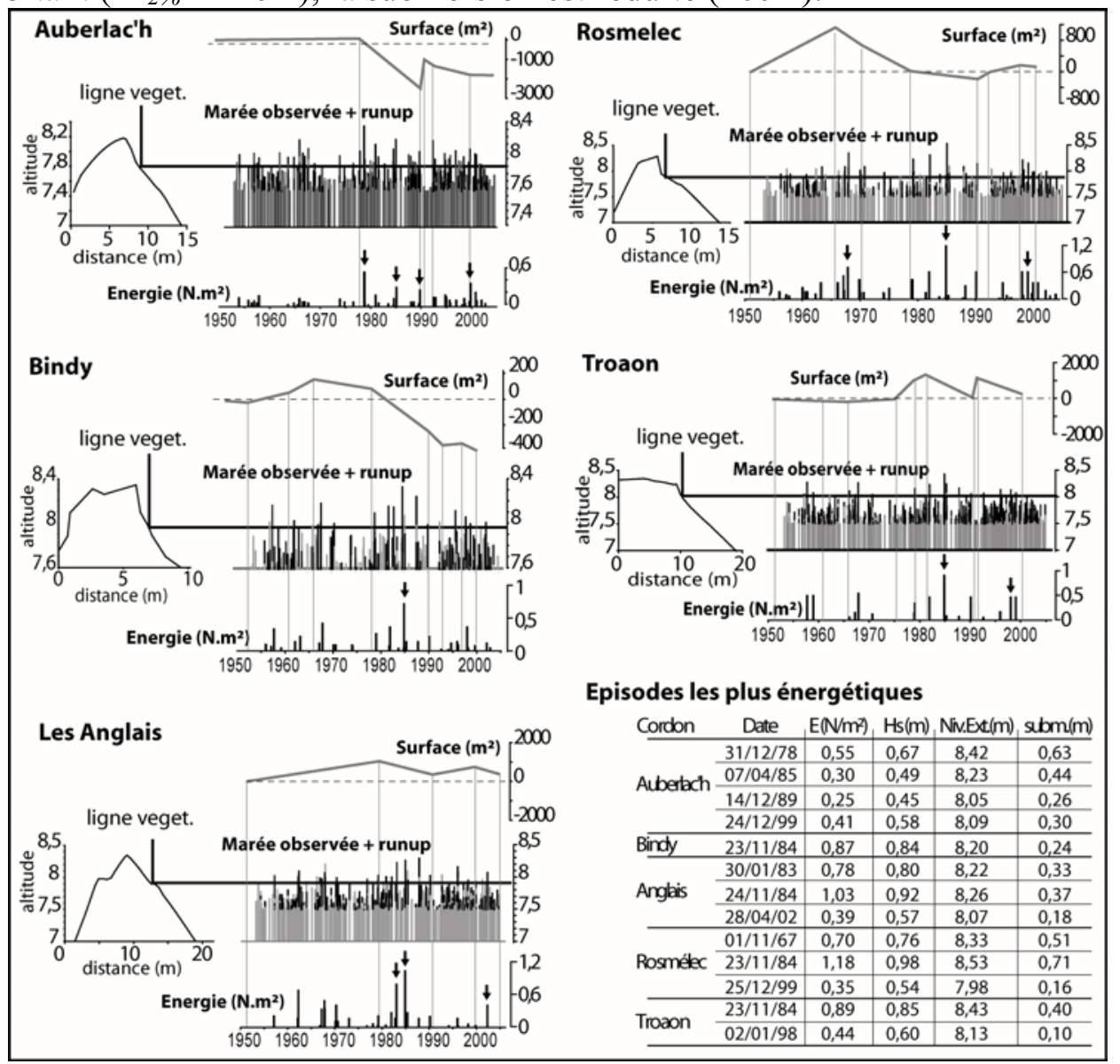

Figure 7 : Evolution morphosédimentaire des cordons littoraux de l'Auberlac'h, Bindy, Rosmélec, Troaon, les Anglais et conditions de forçage météo-marines associées.

\section{Conclusion}

Cette étude montre que le recul des cordons littoraux en rade de Brest s'effectue principalement lors des périodes marquées par une série d'épisodes de hauts niveaux marins très énergétiques. Les années 1967, 1978, 1983-1984, 1989-1990, 1998 et 2002 apparaissent comme les plus morphogènes des cinquante dernières années, provoquant une importante érosion des cordons et se soldant parfois par l'écrêtement et l'ouverture de brèches au sein des accumulations littorales (Loc'h, Auberlac'h). La réponse morphosédimentaire aux forçages météo-marins est toutefois plus marquée sur les secteurs littoraux exposés à des fetch importants et sur lesquels les épisodes morphogènes sont peu fréquents mais très énergétiques. Outre leur degré d'exposition aux agents de forçages météo-marins, d'autres 
paramètres doivent néanmoins être pris en compte pour mieux cerner le fonctionnement morphosédimentaire de ces cordons, tels que la nature sédimentologique des accumulations, la variabilité des apports sédimentaires à la côte, les perturbations introduites par l'homme dans le système littoral ${ }^{[7]}$.Enfin, au terme de cinquante années d'évolution morphosédimentaire, il apparaît que six des dix cordons littoraux étudiés sont en recul, trois d'entre eux présentent une situation d'équilibre et un seul semble en voie d'engraissement. Il semble donc se dégager une tendance à l'érosion des accumulations littorales en rade de Brest. On peut alors s'interroger sur les causes d'une telle évolution. Est-ce la conséquence de l'accélération de la montée relative du niveau marin à Brest depuis $1980^{[9]}$ ? Doit-on y voir le signe d'une pénurie sédimentaire et d'un appauvrissement des sources d'alimentation des cordons littoraux? Le bilan de cinquante années d'évolution littorale sur les dix secteurs étudiés en rade de Brest met en évidence une fragilité de ces cordons, ce qui pose la question de leur devenir à un moment où émerge un intérêt patrimonial pour ce type de formation littorale.

\section{Bibliographie}

1 Garratt, J.R. (1977). Review of drag coefficients over oceans and continents, Monthly Weather Review, 105, 915-929.

2 Guilcher A., Vallantin P., Angrand J.P., Galloy P., (1957). Les cordons littoraux de la rade de Brest, C.O.E.C, 1, 21-54.

3 Hasselmann, K. et al. (1977). Measurements of wind-wave growth and swell decay during the Joint North Sea Wave Project (JONSWAP), Deutsches Hydrographisches Institut, Suppl. A8, 12, 95p.

4 Jackson N.L., Nordstrom K.F., Eliot I., Masselink G., (2002). "Low energy" sandy beaches in marine and estuarine environments: a review, Geomorphology, 48, 147-162.

5 Komar, P.D. (1998). Beach processes and sedimentation, 2nd Edit., Printice Hall, 544p.

6 Stéphan P., Suanez S., Fichaut B., (2005). Impacts de l'anthropisation sur l'évolution morphosédimentaire d'un système littoral de flèches en chicane, le Sillon de Mengleuz à Logonna-Daoulas en rade de Brest (Finistère), in "Milieux littoraux, Nouvelles perspectives d'étude », Ed. L'Harmattan, Paris, 95-114.

7 Stéphan P., Fichaut B., Suanez S., (2005). Les cordons littoraux de Mengleuz et du Loc'h de Landévennec : aspects récents et actuels de l'érosion de deux flèches de galets en rade de Brest, Bull. Soc. Géol. Minéral. Bretagne, (D), 2, 1-19.

8 Vincent, C.L., Resio, D.T. (1990). Wave forecasting and hindcasting in deep and shallow water, in Handbook of Coastal and Ocean Engineering, 1, Edit. J.B. Herbich, 213-248.

9 Wöppelmann, G., Prouveau, N., Simon, B. (2006). Brest sea level record: a time series construction back to the early eighteenth century, Ocean Dynamics, 56, (sous presse).

10 Young, I.R. (1998). An experiental investigation of the role of atmospheric stability in wind wave growth, Coastal engineering, 34, 23-33. 